\title{
Evaluation of Medicinal Effects of Isoxazole Ring Isosteres on Zonisamide for Autism Treatment by Binding to Potassium Voltage-Gated Channel Subfamily D Member 2 (Kv 4.2)
}

\author{
Mehdi Nabatia,*, Vida Bodaghi-Namileh ${ }^{\mathrm{b}}$
}

${ }^{a}$ Research and Development Department, Shari Pharmaceutical Company, Tehran, Iran

b Department of Toxicology and Pharmacology, Faculty of Pharmacy, Tehran University of Medical Sciences, Tehran, Iran

\section{ART I C L E I N F O}

Received: 19 October 2019

Revised: 13 December 2019

Accepted: 25 December 2019

Available online: 28 December 2019

\section{K E Y W O R D S}

\section{Autism}

Drug design

Molecular docking

Molecular simulation

Potassium channel

Zonisamide

\section{A B S T R A C T}

The present research study discusses discovery of the novel drugs based on Zonisamide (FDA-approved drug) to treat the autism disease. We designed novel compounds by changing the pyrazole ring of the molecular structure with its isosteric rings. The main goal of the present study is evaluation of isosterism effect on Zonisamide compound. The studied pyrazole isosters are isothiazole, [c] azaphosphole, [d] azaphosphole, oxaphosphole, thiaphosphole and diphosphole. First, all designed molecular structures were optimized using density functional theory (DFT) computational method by B3LYP/6$311++G(d, p)$ basis set of theory. All the computations were performed in isolated form at room temperature. Then, making complex of all optimized molecular structures with $A$-type potassium voltage gated subfamily d member 2 (Kv 4.2) was studied. The ligand-receptor complexes energy data showed all designed molecules except (1H-indazol-3-yl)methanesulfonamide interct with channel weakly. The residues Phe 75, Asp 86, Phe 84, and Phe 74 played main role in making complex with (1H-indazol-3-yl)methanesulfonamide. However, the ADME and biological properties of the designed molecules were carried out using swissADME and FAF-Drugs4 web tools. Based on the ligand-channel complexes docking data and biochemical properties of the compounds, the pyrazole pentet ring is a suitable isostere for isoxazole ring in Zonisamide.

\section{GRA P H I C A L A B S T RA C T}

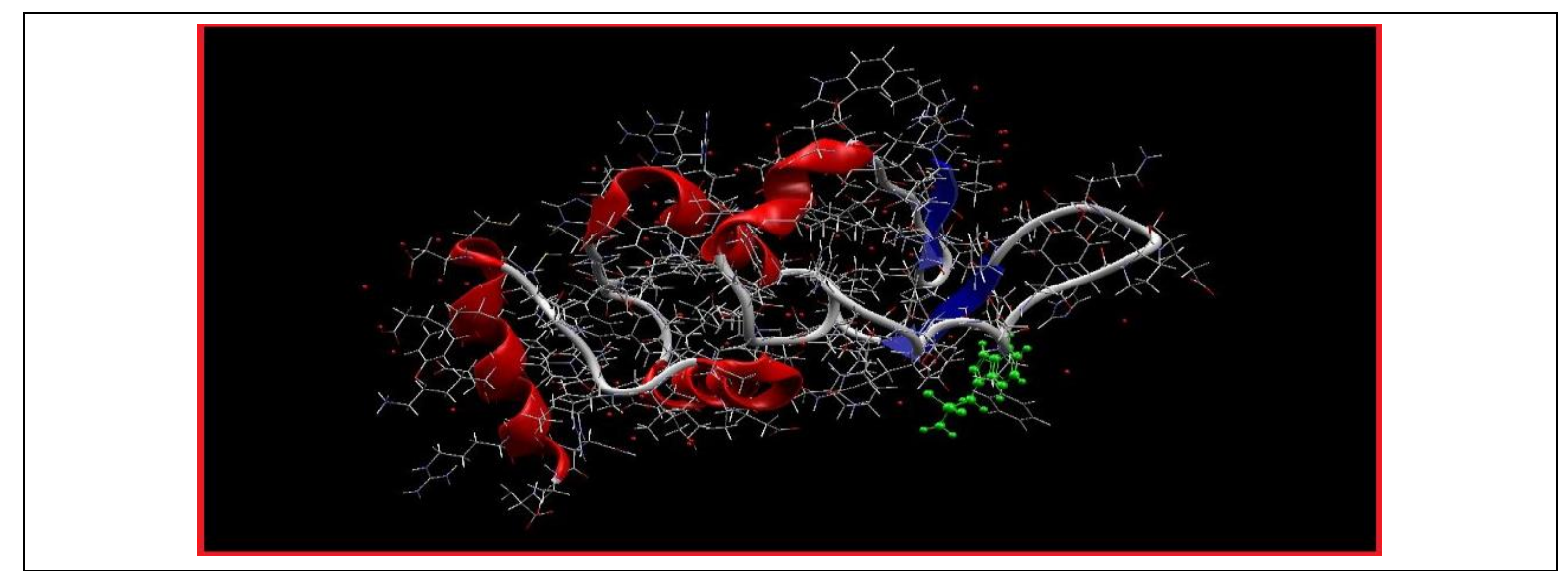

* Corresponding author's E-mail address: mnabati@ymail.com 


\section{Introduction}

Zonisamide or benzo[d]isoxazol-3ylmethanesulfonamide is a sulfonamide, first introduced in 1972 as an antipsychotic drug [1]. Zonisamide later received its FDA approval as an adjuvant treatment for partial seizures in 2000. Over the years, the ameliorative effect of this drug in both partial and generalized seizures [2-7] as well as several other neurological disorders namely, psychotic conditions [8], Parkinson disease [9-13], neuropathic pain [14] and essential tremor [15] have been investigated. Although the precise mechanism of Zonisamide has not yet fully understood, blockage of voltagegated sodium channels and $T$-type calcium channels seems to be the principle route of action. Furthermore, Zonisamide has been observed to interact with $A$-type potassium channels in hippocampal cells and actively block them $[16,17]$. Since the introduction of $A$-type potassium voltage gated subfamily $\mathrm{d}$ member $2(\mathrm{Kv} 4.2)$ as a key player in pathogenesis of Autism spectrum disorders, the use of potassium channel blockers which specifically interact with $\mathrm{Kv} 4.2$ subunit became an area of interest in management of autism [18].

In our previous study, we focused on design and introduction of novel Zonisamide analogs which presented us with the best efficiency and structural interactions with $\mathrm{Kv}$ 4.2 subunits [19]. In this regard, we analyzed the effect of alterations in Zonisamide's backbone and addition of functional groups such as $\mathrm{NH}_{2}, \mathrm{OH}, \mathrm{OCH}_{3}$, and $\mathrm{COOH}$ in drug-receptor docking properties. In addition, biological behavior of the investigated compounds was predicted using SwissADME and FAF-drugs4. Based on the gathered results, $\mathrm{P}_{3} \mathrm{TZ}, \mathrm{H}_{2} \mathrm{P}_{3} \mathrm{TZ}$, $\mathrm{M}_{2} \mathrm{P}_{3} \mathrm{TZ}, \quad \mathrm{H}_{4} \mathrm{P}_{3} \mathrm{TZ}$ and $\mathrm{M}_{4} \mathrm{P}_{3} \mathrm{TZ}$ as novel molecular structures with the optimum efficiency and pharmacokinetic attributes were introduced [19].
The objective of the present study is to further investigate alterations of Zonisamide in relation to its capacity in blockade of $\mathrm{Kv} 4.2$. For this purpose, we designed and assessed different isosteres of the lead compound. The concept behind isosterism is to substitute molecules with atoms, ions or molecules that possess the same atom number and valence electrons, leaving back-bone and functional groups intact. In this study, 7 new analogs containing isothiazole, pyrazole, [c] azaphosphole, [d] azaphosphole, oxaphosphole, thiaphosphole and diphosphole were designed, investigated and compared with the base compound. The efficiency and affinity towards Kv 4.2 subunit were evaluated using molecular docking methods and computational chemistry. In addition, the physicochemical and biological attributes of each compound was predicted using SwissADME and FAF-drugs4 web tools.

\section{Experimental}

\section{Computational methods}

Design of novel drugs is a new field of chemistry to introduce the new molecular structures as a medicine based on their interactions with a biologically receptor [20$22]$. These new medicines are commonly the organic small molecules that inhibit, activating the specific biomolecules [23-26]. The results of these interactions make therapeutic effects on the patients [26-28]. A drug design process comprises two main parts: molecular simulation and molecular docking [29]. Simulation of small molecules is done using quantum mechanical (QM) equations solving [30]. Molecular docking is analysis of the interactions of the ligandbiomolecule complex [31]. Here, the molecular structures of all designed chemical compounds based on the medicinal molecule Zonisamide are optimized using density functional theory (DFT) computational method. In first step, optimization of all 
molecular structures are carried out using B3LYP/6-311++G(d,p) level of theory in isolated form at room temperature. We use the Gaussian 03 software for our computations. In second step, all optimized molecular structures are embedded into the potassium voltage-gated channel subfamily D member 2 (Kv 4.2) and the ligandbiomolecule docking is analyzed. It is necessary to say that the crystal structure of the title potassium channel was taken from the PDB (https://www.rcsb.org/). The Molegro Virtual Docker (MVD) software is used to the molecular docking analyses. On the other hand, the physicochemical properties of the studied compounds are gained using the online web tool www.swissadme.ca and discussed. The druglikeness properties are analyzed using the FAF-Drugs4 server.

\section{Results and discussions}

Molecular docking analysis of the designed compounds binding to the potassium voltagegated channel subfamily D member 2 (Kv 4.2)

As mentioned above, we studied the electronic properties, stability and reactivity of Zonisamide in our previous article. We showed the B3LYP/6-311++G(d,p) basis set of theory is the best computational method to study this compound and its analogues [19]. So, this computational method will be used to optimize our new designed compounds. Scheme 1 shows the molecular structure of the novel designed molecules.

It was said Zonisamide can make complex with the potassium voltage-gated channel $\mathrm{Kv}$ 4.2. As seen in Scheme 1, new compounds were designed based on changing the isoxazole pentet ring to its isosteres. To attain the study of possibility of compounds interaction with the title receptor, all molecular structures were optimized at B3LYP/6-311++G(d,p) level of theory. Then, the optimized molecular structures were docked into the potassium voltage-gated channel Kv 4.2. Table 1 indicates the molecular structures-receptor docking data. We can see all new compounds except the compound containing pyrazole ring show low interaction energy with the channel. So, only the (1H-indazol-3-yl)methanesulfonamide can make complex with the channel as well as Zonisamide.

The interactions of the Zonisamide optimized molecular structure with the potassium voltage-gated channel subfamily $\mathrm{D}$ member $2(\mathrm{Kv} 4.2)$ were analyzed in our previous research work [19]. Here, we want to evaluate docking of (1H-indazol-3yl)methanesulfonamide analog into the title receptor (Figure 1). As seen from the data of Table 1, the Mol Dock total energy score of the compound binding to the channel is -81.6029 .
Scheme 1. The designed molecular structures based on Zonisamide

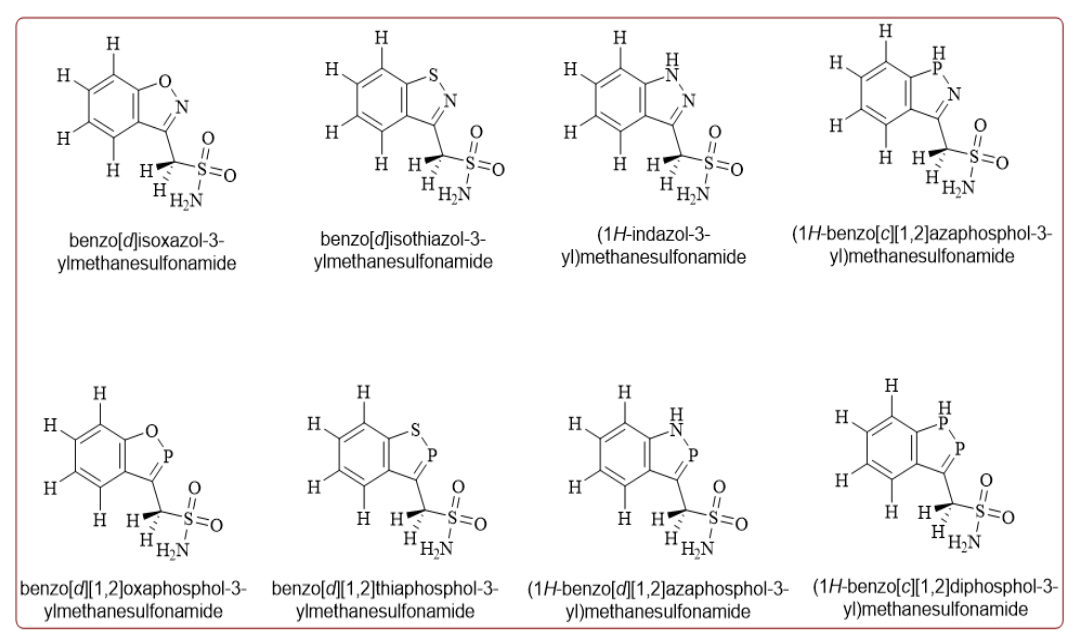


Table 1. The molecular structures-receptor docking analysis data

\begin{tabular}{cc} 
Compound & Mol Dock Score \\
Zonisamide or benzo[d]isoxazol-3-ylmethanesulfonamide & -81.8229 \\
benzo[d]isothiazol-3-ylmethanesulfonamide & -74.2030 \\
(1H-indazol-3-yl)methanesulfonamide & -81.6029 \\
(1H-benzo[c][1,2] azaphosphol-3-yl)methanesulfonamide & -70.0314 \\
benzo[d][1,2] oxaphosphol-3-ylmethanesulfonamide & -70.7224 \\
benzo[d][1,2] thiaphosphol-3-ylmethanesulfonamide & -68.6312 \\
(1H-benzo[d][1,2]azaphosphol-3-yl)methanesulfonamide & -71.7170 \\
(1H-benzo[c][1,2]diphosphol-3-yl)methanesulfonamide & -62.6987 \\
\hline
\end{tabular}

Figure 1. Ligands Zonisamide and $(1 \mathrm{H}-$ indazol-3-yl)methanesulfonamide embedded in the active site of the potassium voltage-gated channel Kv 4.2

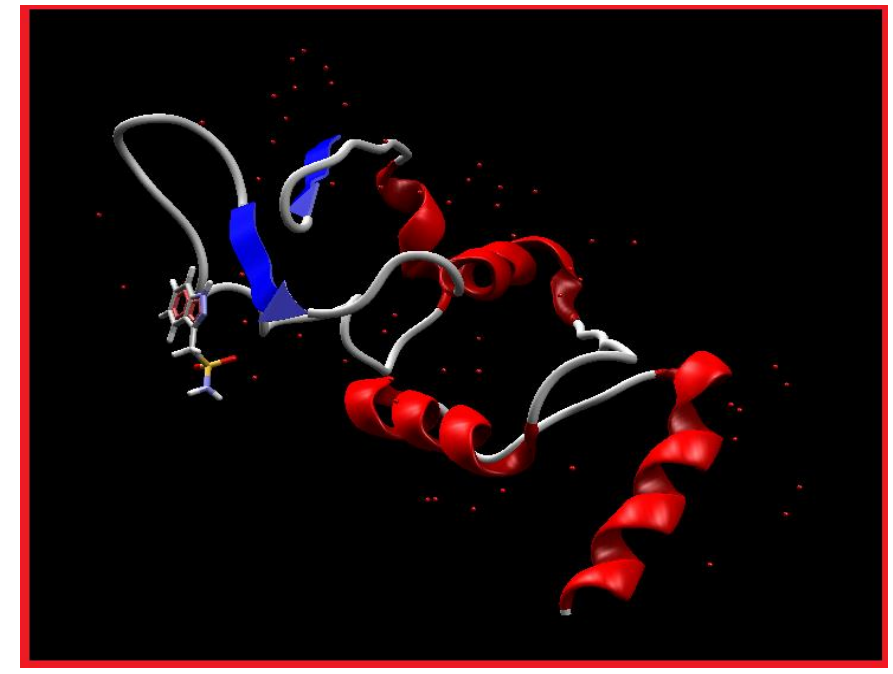

The steric interactions (score $=-65.510$ ), water-ligand interactions (score $=-15.985$ ) and hydrogen bond interactions (score $=-0.195$ ) cause binding the drug to the channel. So, the steric interactions play main role at making complex between drug and receptor. These steric interactions are carried out with residues Phe 75, Asp 86, Phe 84, Phe 74, Phe 85, His 77, Phe 121, Tyr 76, and Arg 87. In overall, the residues Phe 75, Asp 86, Phe 84, and Phe 74 play main role in making the ligand-receptor complex.

Physicochemical descriptors and ADME parameters of the designed compounds

The prediction of physiochemical descriptors and ADME behavior of molecular compounds is considered a predominant factor in early stages of drug discovery [32-35]. SwissADME web tool was used for prediction and computed analysis of ADME properties and the results are presented in Tables 2 and 3 .
Molecular formula, molecular weight, the number of heavy atoms and aromatic heavy atoms, fraction csp3, the number of rotatable bond, $H$-bond receptors and $H$-bond donors, molar refractivity and TPSA (Topological Polar Surface Area) are calculated and presented in physiochemical properties section. Lipophilicity is of great importance in areas of drug discovery and design as it affects several attributes of the compound such as membrane permeability, solubility, selectivity, toxicity and potency. Lipophilicity values are presented as the partition coefficient between $n$-octanol and water $(\log \mathrm{PO} / \mathrm{W})$. Five predictive models (iLOGP, XLOGP, WLOGP, MLOGP and SILICOSIT) are utilized in SwissADME to analyze compound's lipophilicity. The lipophilicity profile of investigated compounds were somewhat similar with MLOGP values of -0.46 in Zonisamide and (1H-indazol-3-yl)methanesulfonamide, -0.44 in benzo[d]isothiazol-3-ylmethanesulfonamide, 0.71 in (1H-benzo[c][1,2]azaphosphol-3- 
yl)methanesulfonamide, $-0.36$

in benzo[d][1,2] oxaphosphol-3ylmethanesulfonamide and benzo[d][1,2]azaphosphol-3-

yl)methanesulfonamide, 0.49 benzo[d]isothiazol-3-ylmethanesulfonamide and finally 0.22 in $(1 \mathrm{H}-$ benzo[c] [1,2]diphosphol3-yl)methanesulfonamide. The next factor which significantly influences drug bioavailability and absorption from Gastrointestinal track (GIT) is Water solubility. Oral pharmaceutical formulations are especially reliant on this factor for their optimal concentration in systemic circulation. Water solubility of each investigated compound was determined using ESOL model, a topical method to evaluate Log S. In this regard, compounds are generally categorized in six groups: Insoluble (Log $\mathrm{S}<-10$ ), poorly soluble $(-10<\log \mathrm{S}<-6)$, moderately soluble $(-6<\log \mathrm{S}<-4)$, soluble $(-4<\log \mathrm{S}<-2)$, very soluble $(-2<\log S<0)$ and highly soluble $(\log S>$ $0)$. As observed in Tables 2 and 3, all substitutions fall into soluble and very soluble category. Individual ADME behaviors of the molecules under investigation are evaluated in pharmacokinetics section which evaluates the compounds passive gastrointestinal (GI) absorption, Blood Brain Barrier (BBB) permeability, $P$-gp efflux, inhibition of Cytochrome P450 enzymes (CYP1A2, CYP2C19, CYP2C9, CYP2D6 and CYP3A4). GI absorption is presented as high and low values. All compounds displayed high GI absorptions. Furthermore, none of the investigated structures are BBB permeant and P-gp efflux pump substrates. Cytochrome P450 enzymes are key players in the process of drug's metabolic biotransformation and further elimination.

Table 2. ADME properties of Zonisamide, benzo[d]isothiazol-3-ylmethanesulfonamide, $(1 \mathrm{H}-$ indazol-3-yl)methanesulfonamide, and (1H-benzo[c][1,2]azaphosphol-3yl)methanesulfonamide

\begin{tabular}{|c|c|c|c|c|}
\hline \multicolumn{5}{|c|}{ Compounds } \\
\hline $\begin{array}{c}\text { Physiochemical } \\
\text { descriptors and ADME } \\
\text { parameters }\end{array}$ & Zonisamide & $\begin{array}{l}\text { benzo[d]isothiazol-3- } \\
\text { ylmethanesulfonamide }\end{array}$ & $\begin{array}{l}\text { (1H-indazol-3- } \\
\text { yl)methanesulfonamide }\end{array}$ & $\begin{array}{c}(1 \mathrm{H}- \\
\text { benzo[c][1,2]azaphosphol- } \\
\text { 3-yl)methanesulfonamide }\end{array}$ \\
\hline Formula & $\mathrm{C}_{8} \mathrm{H}_{8} \mathrm{~N}_{2} \mathrm{O}_{3} \mathrm{~S}$ & $\mathrm{C}_{8} \mathrm{H}_{8} \mathrm{~N}_{2} \mathrm{O}_{2} \mathrm{~S}_{2}$ & $\mathrm{C}_{8} \mathrm{H}_{9} \mathrm{~N}_{3} \mathrm{O}_{2} \mathrm{~S}$ & $\mathrm{C}_{8} \mathrm{H}_{9} \mathrm{~N}_{2} \mathrm{O}_{2} \mathrm{PS}$ \\
\hline MW (g/mol) & 212.23 & 228.29 & 211.24 & 228.21 \\
\hline Num. Heavy Atoms & 14 & 14 & 14 & 14 \\
\hline $\begin{array}{l}\text { Num. Arom. Heavy } \\
\text { Atoms }\end{array}$ & 9 & 9 & 9 & 9 \\
\hline Fraction Csp3 & 0.12 & 0.12 & 0.12 & 0.12 \\
\hline $\begin{array}{l}\text { Num. Rotatable } \\
\text { bonds }\end{array}$ & 2 & 2 & 2 & 2 \\
\hline $\begin{array}{l}\text { Num. } H \text {-bond } \\
\text { acceptors }\end{array}$ & 5 & 4 & 4 & 4 \\
\hline Num. $H$-bond donors & 1 & 1 & 2 & 1 \\
\hline Molar Refractivity & 50.65 & 56.26 & 52.73 & 57.88 \\
\hline TPSA $\left(\AA^{2}\right)$ & 95.57 & 109.67 & 97.22 & 81.43 \\
\hline MLOGP & -0.46 & -0.44 & -0.46 & -0.71 \\
\hline Log S (ESOL) & -1.65 & -2.14 & -1.55 & -1.62 \\
\hline Class & Very Soluble & Soluble & Soluble & Soluble \\
\hline GI Absorption & High & High & High & High \\
\hline BBB permeant & No & No & No & No \\
\hline$P$-gp substrate & No & No & No & No \\
\hline PYP450 inhibitor & No & No & No & No \\
\hline Lipinski & Yes & Yes & Yes & Yes \\
\hline Bioavailability Score & 0.55 & 0.55 & 0.55 & 0.56 \\
\hline
\end{tabular}


Table 3. ADME properties of benzo[d][1,2]oxaphosphol-3-ylmethanesulfonamide, benzo[d][1,2]thiaphosphol-3-ylmethanesulfonamide, (1H-benzo[d][1,2]azaphosphol-3yl)methanesulfonamide and (1H-benzo[c][1,2]diphosphol-3-yl)methanesulfonamide

\begin{tabular}{|c|c|c|c|c|}
\hline \multicolumn{5}{|c|}{ Compounds } \\
\hline $\begin{array}{c}\text { Physiochemical } \\
\text { descriptors and } \\
\text { ADME parameters }\end{array}$ & $\begin{array}{l}\text { benzo[d][1,2]o } \\
\text { xaphosphol-3- } \\
\text { ylmethanesulf } \\
\text { onamide }\end{array}$ & $\begin{array}{l}\text { benzo[d][1,2]thiaphosphol } \\
\text {-3-ylmethanesulfonamide }\end{array}$ & $\begin{array}{c}(1 \mathrm{H}- \\
\text { benzo[d][1,2]azaphosphol } \\
\text {-3-yl)methanesulfonamide }\end{array}$ & $\begin{array}{l}\text { (1H-benzo[c][1,2]diphosphol-3- } \\
\text { yl)methanesulfonamide }\end{array}$ \\
\hline Formula & $\mathrm{C}_{8} \mathrm{H}_{8} \mathrm{NO}_{3} \mathrm{PS}$ & $\mathrm{C}_{8} \mathrm{H}_{8} \mathrm{NO}_{2} \mathrm{PS}_{2}$ & $\mathrm{C}_{8} \mathrm{H}_{9} \mathrm{~N}_{2} \mathrm{O}_{2} \mathrm{PS}$ & $\mathrm{C}_{8} \mathrm{H}_{9} \mathrm{NO}_{2} \mathrm{P}_{2} \mathrm{~S}$ \\
\hline $\mathrm{MW}(\mathrm{g} / \mathrm{mol})$ & 229.19 & 245.26 & 228.21 & 245.18 \\
\hline $\begin{array}{l}\text { Num. Heavy } \\
\text { Atoms }\end{array}$ & 14 & 14 & 14 & 14 \\
\hline $\begin{array}{l}\text { Num. Arom. } \\
\text { Heavy Atoms }\end{array}$ & 6 & 6 & 6 & 6 \\
\hline Fraction Csp3 & 0.12 & 0.12 & 0.12 & 0.12 \\
\hline $\begin{array}{l}\text { Num. Rotatable } \\
\text { bonds }\end{array}$ & 2 & 2 & 2 & 2 \\
\hline $\begin{array}{l}\text { Num. H-bond } \\
\text { acceptors }\end{array}$ & 4 & 3 & 3 & 3 \\
\hline $\begin{array}{l}\text { Num. } H \text {-bond } \\
\text { donors }\end{array}$ & 1 & 1 & 2 & 1 \\
\hline Molar Refractivity & 55.63 & 60.86 & 61.38 & 62.96 \\
\hline TPSA $\left(\AA^{2}\right)$ & 111.91 & 127.98 & 114.71 & 116.27 \\
\hline MLOGP & -0.36 & 0.49 & -0.36 & 0.22 \\
\hline Log S (ESOL) & -1.21 & -1.40 & -1.21 & -1.05 \\
\hline Class & Very soluble & Very soluble & Very soluble & Very soluble \\
\hline GI Absorption & High & High & High & High \\
\hline BBB permeant & No & No & No & No \\
\hline$P$-gp substrate & No & No & No & No \\
\hline PYP450 inhibitor & No & No & No & No \\
\hline Lipinski & Yes & Yes & Yes & Yes \\
\hline $\begin{array}{l}\text { Bioavailability } \\
\text { score }\end{array}$ & 0.55 & 0.55 & 0.55 & 0.56 \\
\hline
\end{tabular}

As a result, the evaluation of inhibitory capacity of compounds is essential in order to predict their possible interactions with other drugs and adverse effects resulting from decreased clearance and accumulation of the drug or its metabolites. Neither of the mentioned compounds inhibits cytochrome P450 enzymes. Lipinski's rule of five and bioavailability score of the investigated compounds determines their drug-likeness. All 8 compounds abide Lipinski's rule (MLOGP $\leq 4.15$, relative $\mathrm{MW} \leq 500$, $\mathrm{N}$ or $0 \leq 10$, $\mathrm{NH}$ or $\mathrm{OH} \leq 5)$. Bioavailability score is used to forecast the permeability and bioavailability properties of a compound in discovery setting. The values are 0.55 in all investigated compounds.

The molecular structures were further analyzed using the FAF-drugs4 web tool.
The results are presented in Figures 2-7. The Figure 2 is Physchem Filter Positioning which provides a radar plot, incorporating all predicted physiochemical descriptors. The compound's values (blue line) should reside in the drug-like filter area (pale blue and red). As observed in Figure 2, all compounds fall within the designated ranges.

Figure 3 visualizes the compound complexity. It involves the number of system ring, stereo centers, rotatable and rigid bonds, the flexibility (ration between rotatable and rigid bonds), the carbon saturation (fsp3 ratio) and the maximum size of system rings. The values for all 8 compounds (blue line) are superimposed on the oral library min and max ranges (determined by red and pink areas). 

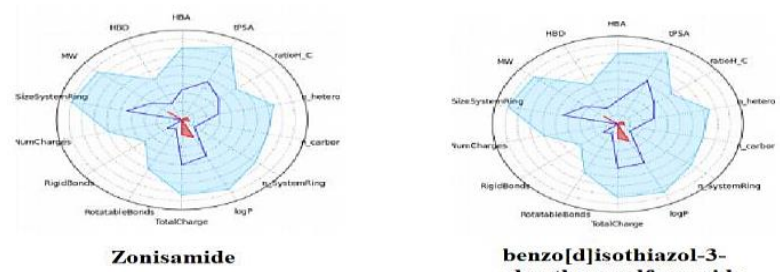

benzo[d]isothiazol-3ylmethanesulfonamide

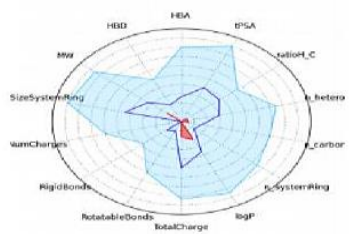

(1H-benzo[c][1,2] azaphosphol-3yl)methanesulfonamide

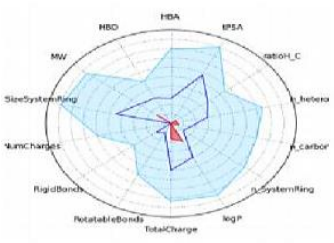

(1H-benzo[d][1,2]azaphosphol-3yl)methanesulfonamide
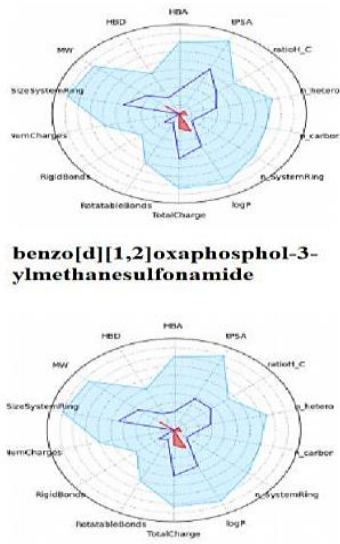

(1H-benzo[c][1,2]diphosphol-3yl)methanesulfonamide

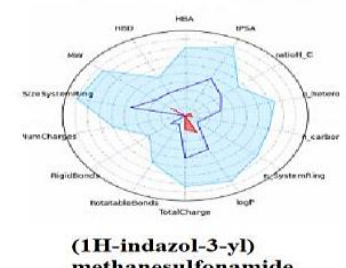

(1H-indazol-3-yl)
methanesulfonamide

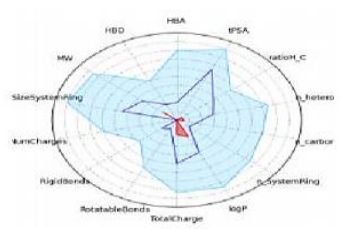

benzo[d][1,2]thiaphosphol-3ylmethanesulfonamide

Figure 2. PhysChem Filter Positioning of the designed compounds

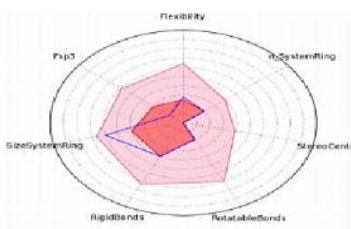

Zonisamide

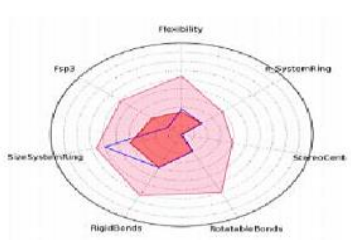

(1H-benzo[c][1,2] azaphosphol-3yl)methanesulfonamide

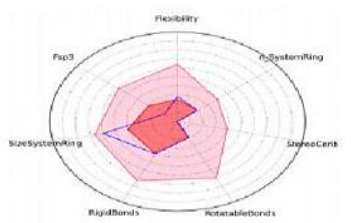

(1H-benzo[d] [1,2] azaphosphol-3yl)methanesulfonamide

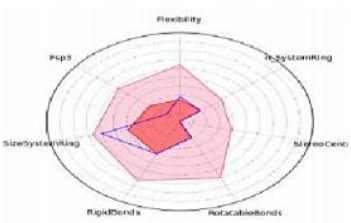

benzo[d]isothiazol-3 ylmethanesulfonamide

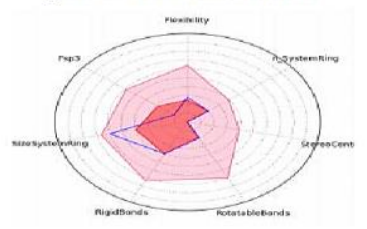

benzo $[d][1,2]$ oxaphosphol-3 ylmethanesulfonamide

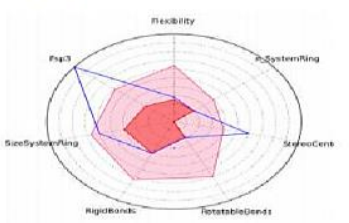

(1H-benzo[c] [1,2]diphosphol-3yl)methanesulfonamide

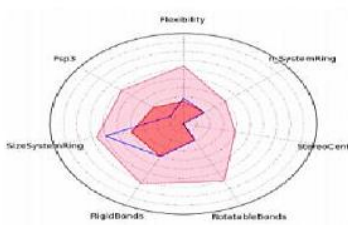

(1H-indazol-3-yl) (1H-indazol-3-yl)

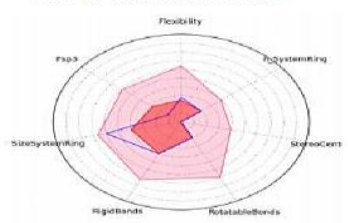

benzo $[d][1,2]$ thiaphosphol-3 ylmethanesulfonamide

Figure 3. Compound Complexity of the designed compounds

Figure 4 analyses Golden Triangle Rule which is a visualization tool used to optimize clearance and oral absorption of drug candidates. The compounds located in the triangle are likely to have an optimal permeability (low clearance) and a good metabolic stability. As presented in Figure 4, all compounds are positioned inside the golden triangle. 
Figure 5 represents the Oral Property Space, which is obtained by applying the PCA (Principal Component Analysis) of the 15 main physico-chemical descriptors of the chosen compounds (red), compared with two oral libraries extracted from eDrugs (blue) and DrugBank (orange). All compounds are located within the specified range.

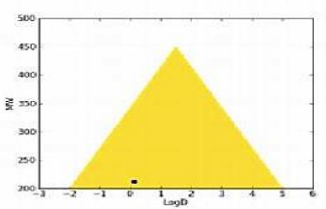

Zonisamide

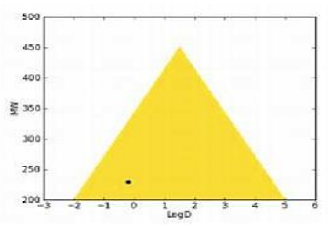

(1H-benzo[c][1,2]azaphosphol-3yl)methanesulfonamide

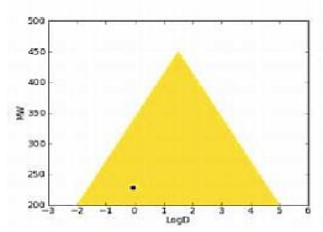

(1H-benzo[d][1,2] azaphosphol-3yl)methanesulfonamide
Oral Absorption Estimation is presented in Figure 6. The compounds values are represented by the blue line, which should fall within the optimal green area (Rule of 5 and Verber rule area). The white area is the extreme maximum zone and the red one is the extreme minimum zone.

Figure 4. Golden Triangle Rule for the designed compounds

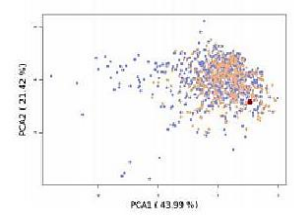

Zonisamide

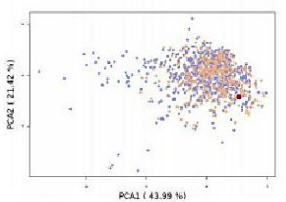

(1H-benzo[c][1,2] azaphosphol-3yl)methanesulfonamide

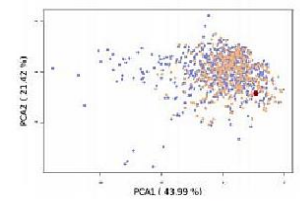

(1H-benzo[d][1,2] azaphosphol-3yl)methanesulfonamide

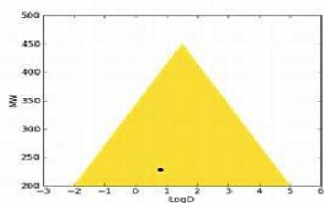

benzo [d] isothiazol-3ylmethanesulfonamide

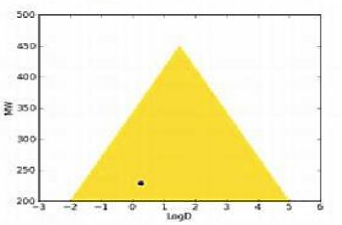

benzo[d][1,2]oxaphosphol-3ylmethanesulfonamide

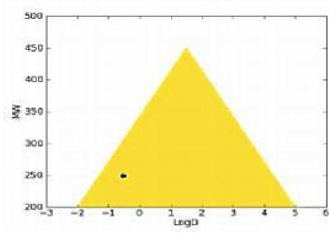

(1H-benzo[c][1,2] diphosphol-3 yl)methanesulfonamide

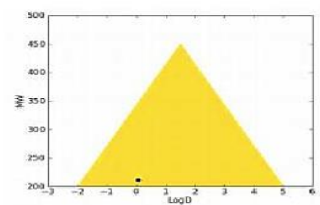

(1H-indazol-3-yl) methanesulfonamide

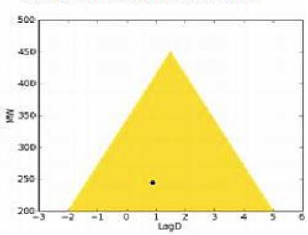

benzo $[d][1,2]$ thiaphosphol-3ylmethanesulfonamide

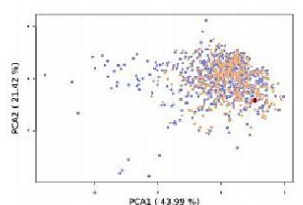

benzo[d]isothiazol-3ylmethanesulfonamide

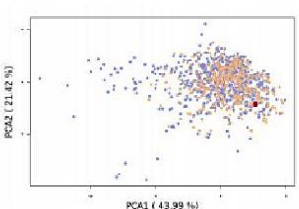

benzo[d][1,2] oxaphosphol-3ylmethanesulfonamide

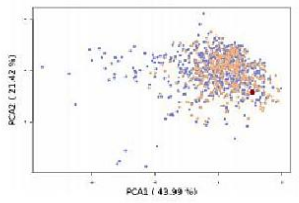

(1H-benzo[c][1,2]diphosphol-3yl)methanesulfonamide

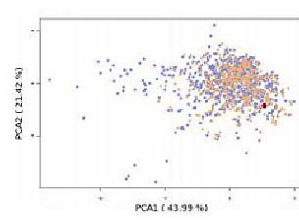

(1H-indazol-3-yl) methanesulfonamide

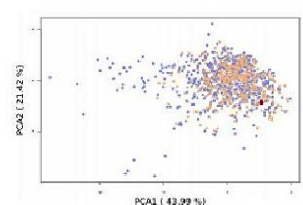

benzo[d] [1,2]thiaphosphol-3 ylmethanesulfonamide

Figure 5. Oral Property Space of the designed compounds 
These zones are determined by the following descriptors ranges: LOGP ( -2 to 5$)$, MW (150 to 500), tPSA (20 to 150), Rotatable Bonds (0 to 10), $H$-Bonds Acceptors ( 0 to 10 ) and Donors ( 0 to 5). All 8 compounds are located within acceptable ranges.

Lastly, Pfizer 3/75 rule is exhibited in Figure 7. Molecules located in red square are more likely to cause toxicity. The compounds under investigation are all placed in the green square predicting them to be non-toxic. Regarding the obtained data from predictive models, all 7 analogs exhibited satisfactory physiochemical properties and biological behaviors same as the base compound (Zonisamide). However, as discussed in the previous section, only $(1 \mathrm{H}$ indazol-3-yl)methanesulfonamide possessed a similar docking capability to Zonisamide while other structures did not show acceptable interactions with $\mathrm{Kv} 4.2$ receptor. In conclusion, considering both biological and docking results, Pyrazole pentet ring in (1H-indazol-3yl)methanesulfonamide could be introduced as a suitable isostere for Isoxazole pentet ring in Zonisamide.

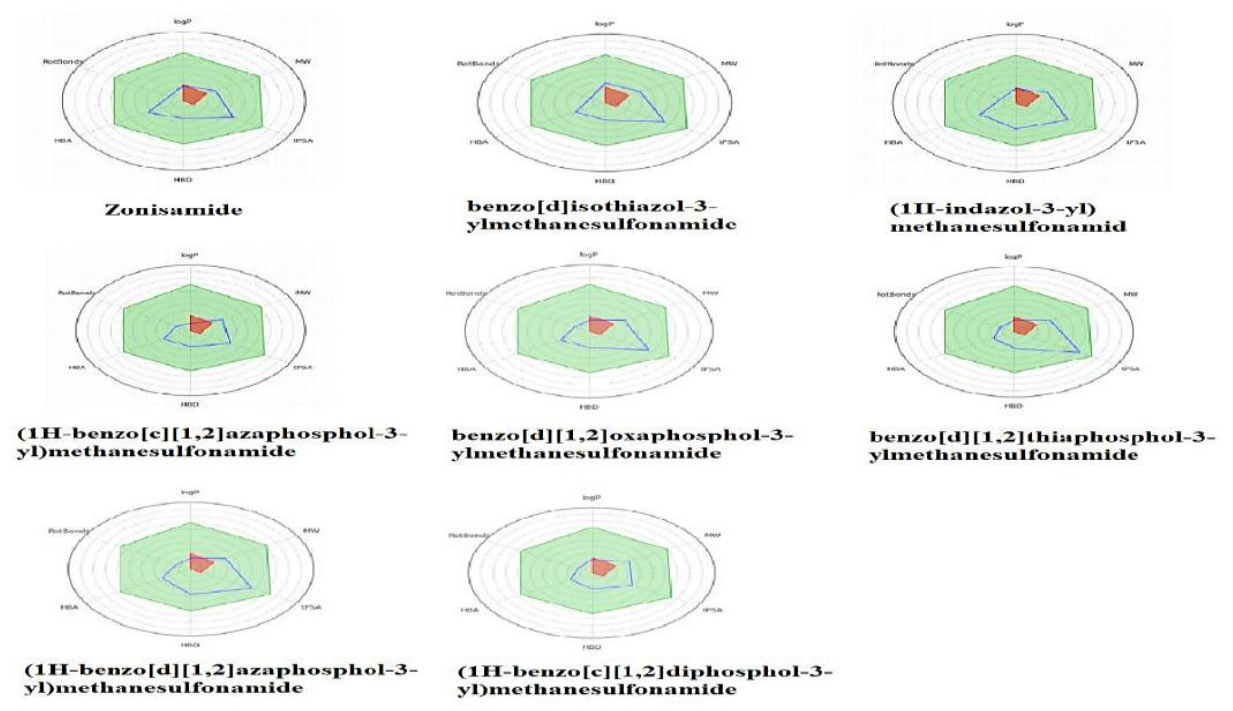

Figure 6. Oral Absorption Estimation of the designed compounds

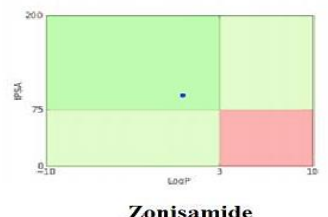

Zonisamide

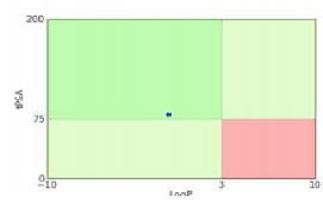

(1H-benzo[c] [1,2]azaphosphol-3yl)methan esulfonamide

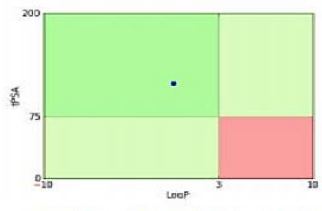

(1H-benzo[d] [1,2] azaphosphol-3yl)methanesulfonamide

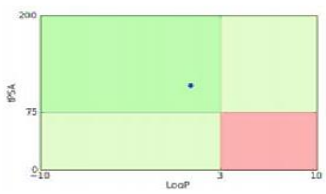

benzo[d] isothiazol-3ylmethanesulfonamide

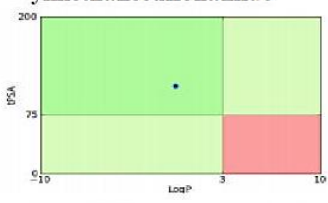

benzo[d] [1,2]oxaphosphol-3ylmethanesulfonamide

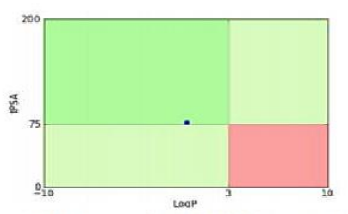

(1H-benzo[c] [1,2]diphosphol-3yl)methanesulfonamide
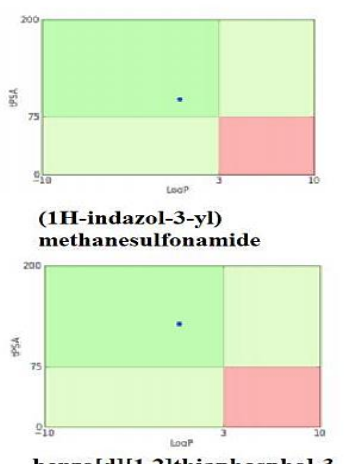

benzo[d] $[1,2]$ thiaphosphol-3 ylmethanesulfonamide

Figure 7. Pfizer $3 / 75$ rule of the designed compounds 


\section{Conclusion}

Zonisamide is a FDA-approved drug for treatment of the epilepsy and autism. In this work, we surveyed the novel compounds based on the Zonisamide by changing the pentet ring of the molecular structure with its isosteric rings. So, we designed 7 new analogs containing isothiazole, pyrazole, [c] azaphosphole, [d] azaphosphole, oxaphosphole, thiaphosphole and diphosphole rings. First, we optimized all molecular structures using density functional theory (DFT) computational method by functional B3LYP and 6-311++G(d,p) level of theory. After that, the optimized molecular structures were interacted with the said potassium channel using molecular docking technique. The docking analysis data showed all designed compounds except $1 \mathrm{H}$-indazol3-yl)methanesulfonamide have weaker interaction with channel than Zonisamide. Prediction of the designed compounds' biochemical properties was performed using the swissADME and FAF-Drugs4 websites. In overall, both the biological and docking results revealed that, Pyrazole pentet ring in (1H-indazol-3-yl)methanesulfonamide could be introduced as a suitable isostere for Isoxazole pentet ring in Zonisamide. We strongly believe that this method of Zonisamide-based compound screening and evaluation will be of great interest in future endeavors in drug design and drug delivery.

\section{Acknowledgment}

The authors are grateful to Dr. Elham Pournamdari for providing invaluable support in this research study.

\section{Disclosure statement}

No potential conflict of interest was reported by the authors.

\section{References}

[1] S.I. Ueno, S. Saiki, M. Fujimaki, H. Takeshige-Amano, T. Hatano, G. Oyama, K.I. Ishikawa, A. Yamaguchi, S. Nojiri, W. Akamatsu, N. Hattori, Cells., 2018, 8, 14.

[2] S.Y. Kwan, Y.C. Chuang, C.W. Huang, T.C. Chen, S.B. Jou, A. Dash, CNS. Neurosci. Ther., 2015, 21, 683-691.

[3] F. Brigo, S. Lattanzi, S.C. Igwe, M. Behzadifar, N.L. Bragazzi, Cochrane Database Syst. Rev., 2018, 10, CD001416.

[4] A. Reimers, H. Ljung, Expert. Opin. Pharmacother., 2019, 20, 909-915.

[5] M. Maschio, L. Dinapoli, A. Zarabla, A. Maialetti, D. Giannarelli, A. Fabi, A. Vidiri, T. Cantelmi, Clin. Neuropharmacol., 2017, 40, 113-119.

[6] A. Romigi, E.A. Femia, C. Fattore, G. Vitrani, G. Di Gennaro, V. Franco, Clin. Interv. Aging., 2015, 10, 931-937.

[7] A. Schulze-Bonhage, Expert. Rev. Neurother., 2015, 15, 857-865.

[8] M. Murata, T. Odawara, K. Hasegawa, S. Iiyama, M. Nakamura, M. Tagawa, K. Kosaka, Neurology, 2018, 90, e664-e672.

[9] K. Ikeda, M. Yanagihashi, K. Miura, Y. Ishikawa, T. Hirayama, T. Takazawa, 0. Kano, K. Kawabe, N. Mizumura, Y. Iwasaki, J. Neurol. Sci., 2018, 391, 5-9.

[10] S. Matsunaga, T. Kishi, N. Iwata, J. Alzheimers. Dis., 2017, 56, 1229-1239.

[11] Y. Fang, F. Mao, M. Zhu, X. Li, Medicine (Baltimore), 2019, 98, e14228.

[12] M. Hirayama, T. Minato, T. Maeda, T. Nakamura, M. Katsuno, Neurol. Clin. Neurosci., 2017, 5, 49-54.

[13] H. Iwaki, M. Tagawa, K. Iwasaki, K. Kawakami, M. Nomoto, J. Neurol. Sci., 2019, 402, 145-152.

[14] M. Nabati, Iran. J. Org. Chem., 2018, 10, 2291-2299.

[15] M. Nabati, Iran. J. Org. Chem., 2018, 10, 2409-2416.

[16] J.C. Martínez-Ávila, A. García Bartolomé, I. García, I, Dapía, H.Y. Tong, L. Díaz, P. 
Guerra, J. Frías, A.J. Carcás Sansuan, A.M. Borobia, Metabolomics., 2018, 14, 70.

[17] A.A. Wilfong, J.L. Jr Holder, Expert. Opin. Pharmacother., 2011, 12, 2573-2581.

[18] L. Guglielmi, I. Servettini, M. Caramia, L. Catacuzzeno, F. Franciolini, M.C. D'Adamo, M. Pessia, Front. Cell. Neurosci., 2015, 9, 34.

[19] M. Nabati, V. Bodaghi-Namileh, Int. J. New. Chem., 2019, 6, 254-276.

[20] M. Nabati, J. Med. Chem. Sci., 2020, 3, 2234.

[21] M. Nabati, Chem. Methodol., 2018, 2, 223238.

[22] M. Nabati, Iran. Chem. Commun., 2019, 7, 324-334.

[23] M. Nabati, H. Sabahnoo, J. Med. Chem. Sci., 2019, 2, 118-125.

[24] M. Nabati, M. Kermanian, H. Mohammadnejad-Mehrabani, H.R. Kafshboran, M. Mehmannavaz, S. Sarshar, Chem. Methodol., 2018, 2, 128-140.

[25] M. Nabati, Asian J. Green Chem., 2019, 3, 258-270.
[26] M. Nabati, J. Phys. Theor. Chem. IAU Iran, 2017, 14, 283-293.

[27] M. Nabati, Chem. Methodol., 2017, 1, 121135.

[28] M. Nabati, J. Phys. Theor. Chem. IAU Iran, 2017, 14, 49-61.

[29] M. Nabati, H. Sabahnoo, E. Lohrasbi, M. Mazidi, Chem. Methodol., 2019, 3, 383397.

[30] M. Nabati, V. Bodaghi-Namileh, Adv. J. Chem. A, 2020, 3, 58-69.

[31] M. Nabati, E. Lohrasbi, H. Sabahnoo, V. Bodaghi-Namileh, M. Mazidi, H. Mohammadnejad-Mehrabani, A. Tavakkoli, A. Gravand, Chem. Methodol., 2020, 4, 19-33.

[32] M. Nabati, V. Bodaghi-Namileh, Iran. Chem. Commun., 2020, 8, 73-58.

[33] M. Nabati, V. Bodaghi-Namileh, Iran. J. Org. Chem., 2019, 11, 2591-2598.

[34] D. Mandal, R. Maity, H. Beg, G. SalgadoMoran, A. Misra, Mol. Phys., 2018, 116, 515-525.

[35] R. Meenakshi, J. Mol. Struct., 2017, 1127, 694-707.

How to cite this manuscript: Mehdi Nabati, Vida Bodaghi-Namileh, Evaluation of Medicinal Effects of Isoxazole Ring Isosteres on Zonisamide for Autism Treatment by Binding to Potassium Voltage-Gated Channel Subfamily D Member 2 (Kv 4.2), Adv. J. Chem. A. 2020. 3(4). 462-472. 